\title{
Correlation and Path Analysis of Physiological and Genetic Traits in Stay Green Rice (Oryza sativa L.) Genotypes
}

\author{
Suman Kumari Nayak ${ }^{1 *}$, R.K. Panda ${ }^{1}$ and S.R. Das ${ }^{2}$ \\ ${ }^{1}$ Department of Plant Physiology at O.U.A.T, Orissa, India \\ ${ }^{2}$ Department of plant breeding and genetics at O.U.A.T, Orissa, India \\ *Corresponding author
}

\begin{abstract}
A B S T R A C T
Stay green trait is reckoned as a favourable and desirable trait which can beacon the path to achieve enhanced yield potential in crops including rice. In this study, the correlation and path analysis for several physiological and genetic traits were carried out using six elite rice genotypes namely Maudamani, Swarna, Pratikshya, Lalat, Mrunalini and a Columbian functional stay green cultivar Fedearroz-50. The physiological efficiency and the genetic aspects of the test cultivars were studied by examining the different yield attributing traits, physiological parameters and biochemical traits in order to sort out the most promising genotype for their possible use in future breeding programs. The correlation study showed positive association of plot yield with grain number, harvest index, net assimilation rate, chlorophyll content and grain yield per plant but is negatively correlated with leaf area index. Path coefficient analysis revealed the maximum direct effect of nitrogen content on grain yield. Based on the conclusions drawn from the different statistical analysis it was divulged that higher panicle length, panicle number, grain number along with improved fertility, higher chlorophyll and carbohydrate content may have contributed towards the enhancement of yield in the promising genotypes.
\end{abstract}

\section{Introduction}

Globally rice is the human food crop of paramount importance as it is the staple food for more than $60 \%$ of the world population. India stands second in the world in rice production. In India rice is grown in an area of 44 million hectares. The country witnessed an era of tremendous growth with threefold increase in the production and productivity of rice. This hike in the production, during the eighties was predominantly by virtue of high yielding varieties. But, the rice production is required to increase further in order to sustain the present level of self-sufficiency. It is therefore, a challenging task as increase in productivity has to come from the declining and degrading resource base in terms of land, 
water and other inputs.

It is quite evident from the various rice improvement programmes undertaken by India as well as International Rice Research Institute that there has not been any momentous improvement in the yield potential of varieties released after IR 8 (Virmani et al., 1993; Khush, 1995; Peng et al., 1999). The yield stagnation is primarily due to large number of unproductive tillers, limited sink size and lodging susceptibility. Scientists around the world are speculating hard to figure out the possible ways so as to increase the yield potential. Among the various approaches suggested, higher photosynthetic rate, slow leaf senescence, increased carbohydrate storage capacity in stems, a greater reproductive sink capacity, an extended grain-filling period and tolerance to photo-inhibition are thought to be the physiological basis of high grain yield (Dingkuhn et al., 1991).

Delay in leaf senescence has been identified as an important component in the genetic improvement of several crops to promote stress tolerance and yield gain (Peigao et al., 2005). The phenotypes with delayed leaf senescence are known as "stay-green". They are being classified into functional and nonfunctional alias cosmetic stay-green. In functional stay-greens, the transition from the carbon capture period to the nitrogen mobilization (senescence) phase of canopy development is delayed, and the senescence syndrome proceeds slowly which will elongate the assimilatory capacity of the canopy thereby contributing to higher yield. Whereas in a cosmetic stay-green, a lesion interferes with an early step in chlorophyll catabolism. The association between staygreen and desirable traits such as greater number of fertile tillers (Ahlawat et al., 2008), higher number of grains per ear (Luche et al., 2013), higher industrial quality (Silva et al., 2004), tolerance to abiotic (Kassahun et al., 2010) and biotic (Joshi et al., 2007) stresses have been reported.

But there are many stay-green materials with poor yielding capacity hence the trait must be combined with excellent plant type. Average size panicles in a stay-green background will not confer maximum yield potential. It is believed that large panicles are required to store the increased production of carbohydrates resulting from stay-green foliage combined with superior plant type. It is suggested that selection for the stay-green trait combined with large, erect flag leaves is a valid and potentially valuable breeding objective for increased yield potential. Conferring it the purpose of present study is to compare and characterize the functional stay green rice in relation to different physiological and quantitative traits of some promising rice cultivars with the objective to examine the nature and magnitude of character association as well as to assess their effect on yield.

\section{Materials and Methods}

The experiment was conducted under normal cultural conditions at the Instructional Farm of Orissa University of Agriculture and Technology (India) during the kharif season of 2017. The experimental design was completely randomized block design with three replicates. The materials under study were transplanted with a spacing of $15 \times 20 \mathrm{~cm}$ in 7 rows plots of $3.75 \mathrm{~m}$ length. A fertilizer dose of $80 \mathrm{~kg} \mathrm{~N}, 40 \mathrm{~kg} \mathrm{P}_{2} \mathrm{O}_{5}$ and $40 \mathrm{~kg} \mathrm{~K} 2 \mathrm{O}$ per hectare was applied as per the scheduled management practices.

\section{Morphological determination}

At maturity plant height along with panicle length, panicle number, flag leaf area, flag leaf angle, second leaf area and second leaf angle were measured. The plant height was measured from the base of the plant to the tip 
of the topmost panicle once the culms cease to elongate. Similarly the panicle length was measured from the ciliate base of the main culm to the tip of the panicle. The area of the flag leaf and the second leaf were calculated by multiplying the area with a correction factor of 0.725. Apart from these a few other yield parameters were also recorded.

\section{Physiological growth parameters}

In order to study the physiological growth parameters, two hills were uprooted at 40 days after sowing and another two at an interval of two weeks. The plant samples were washed clean followed by the separation of the different plant parts. The leaf area was measured by multiplying the apparent leaf area with a constant factor i.e., 0.75 as suggested by Yoshida, 1981. Dry weight of the different plant parts along with other physiological growth parameters were recorded by drying them at a temperature of $80^{\circ} \mathrm{C}$ for 48 hours in a hot air oven.

\section{Biochemical estimation}

Second leaf of the plant from each replication was taken at 40 DAS, 55 DAS, 70 DAS, flowering and physiological maturity stages to estimate the chlorophyll index. $100 \mathrm{mg}$ of fresh leaf sample was taken and cut into small pieces which were then transferred into test tubes containing $80 \%(\mathrm{v} / \mathrm{v})$ acetone solution for 24 hours under dark conditions. The chlorophyll extract was filtered and the absorbance was measured at $652 \mathrm{~nm}$.

The separated and dried leaf and grain samples were ground into fine powder. Nitrogen content was recorded at all the growth stages of the plant. 200mg of the powdered sample was subjected to digestion. $10 \mathrm{ml}$ of the digested sample was poured into the Micro-Kjeldhal flask along with $10 \mathrm{ml}$ of $40 \%$ (w/w) $\mathrm{NaOH}$. Simultaneously, a flask containing $10 \mathrm{ml}$ of $4 \%$ boric acid with $2-3$ drops of mixed indicator was kept under the condenser to absorb the liberated ammonia gas during the distillation process which was then titrated against $0.05 \mathrm{~N} \mathrm{HCl}$ to estimate the total nitrogen content.

$100 \mathrm{mg}$ of powdered straw and grain samples were hydrolysed with $5 \mathrm{ml}$ of $2.5 \mathrm{~N} \mathrm{HCl}$ by keeping in boiling water bath for three hours in order to estimate the carbohydrate content. The samples were neutralized by sodium carbonate. $10 \mathrm{ml}$ of the sample was centrifuged for $10 \mathrm{~min}$ followed by the collection of the supernatant. Standards were prepared and aliquots were taken. $12 \mathrm{ml}$ of anthrone reagent was added to each followed by their heating in the boiling water bath for eight minutes. After cooling the samples and the standards, absorbance was measured at $630 \mathrm{~nm}$ and the total carbohydrate content was estimated from the calibration curve.

\section{Statistical analysis}

Statistical analysis for the observed data was done following Robinson et al., (1951), Johnson et al., (1955) and Al-Jibouri et al., (1958) for correlation coefficient and Dewey and $\mathrm{Lu}$ (1959) for path analysis.

\section{Results and Discussion}

Detailed knowledge about the nature and magnitude of correlation of character pairs facilitate the selection of promising genotypes for the future breeding programs. The results revealed that the plot yield exhibited strong positive association with fertile grains per panicle, harvest index, net assimilation rate, chlorophyll content and grain yield per plant but is negatively associated with leaf area index. Grain yield per plant was positively correlated with fertile grains per panicle, harvest index and chlorophyll content. Both plot yield and grain yield per plant exhibited positive association with number of fertile grains per panicle, fertility percentage, harvest 
index, post flowering photosynthetic contribution to grain yield, net assimilation rate and chlorophyll content, where as both were negatively associated with 100-grain weight, flag leaf area, leaf area index, leaf area duration and relative leaf growth rate. The strong positive association between plot yield and grain yield per plant revealed that selection on the basis of characters contributing grain yield per plant also bears relevance to plot yield.

The positive association of grain yield per plant with harvest index during the present investigation shows that yield is a function of total dry matter and harvest index and yield can be enhanced by increasing the biomass or harvest index or both (Khush and Virk, 2000, Virk et al., 2004)

Both plot yield and grain yield per plant exhibited positive association with harvest index, post-flowering photosynthetic contribution to grain yield, net assimilation rate and chlorophyll content. The positive association among the above mentioned traits clearly indicates that among the various approaches suggested, higher photosynthetic rate, slow leaf senescence, increased carbohydrate storage capacity in stems, greater reproductive sink capacity, an extended grain filling period and tolerance to photo-inhibition are thought to be the physiological basis of high grain yield (Dingkuhn et al., 1991).

It was also observed from the path coefficient analysis that nitrogen content exhibited maximum positive direct effect on grain yield followed by plant height, flag leaf angle, chlorophyll content, $2^{\text {nd }}$ leaf area, leaf weight ratio, leaf area duration, leaf area index, panicle number, net assimilation rate, post flowering photosynthetic contribution to grain yield and $2^{\text {nd }}$ leaf angle.

Table.1 Estimates of phenotypic correlation coefficients among twenty seven characters in rice

\begin{tabular}{|c|c|c|c|c|c|c|c|c|c|c|c|c|c|c|}
\hline SI.No & Character & PH & PL & PN & FGN & $\mathbf{F} \%$ & $\begin{array}{l}\text { 100- } \\
\text { GW }\end{array}$ & FLA & FLAr & 2nd LA & 2nd LAr & HI & PPCG & LAR \\
\hline 1 & FD & 0.159 & -0.452 & -0.207 & 0.305 & 0.573 & -0.504 & 0.252 & 0.404 & -0.175 & 0.274 & -0.247 & 0.608 & -0.576 \\
\hline 2 & PH & & -0.275 & $-0.723^{*}$ & 0.188 & -0.217 & 0.387 & 0.156 & $0.840^{*}$ & -0.430 & $0.724^{*}$ & -0.561 & -0.135 & -0.689 \\
\hline 3 & PL & & & 0.391 & -0.390 & 0.069 & 0.044 & -0.225 & -0.286 & 0.128 & -0.394 & 0.231 & -0.256 & $0.821 *$ \\
\hline 4 & PN & & & & -0.544 & -0.252 & -0.604 & $-0.738^{*}$ & $-0.741^{*}$ & -0.259 & $-0.983 * *$ & 0.266 & -0.401 & 0.773* \\
\hline 5 & FGN & & & & & 0.609 & -0.133 & $0.837^{*}$ & -0.010 & 0.170 & 0.667 & 0.550 & 0.779* & -0.455 \\
\hline 6 & F\% & & & & & & -0.285 & $0.737^{*}$ & -0.021 & 0.470 & 0.339 & 0.426 & $0.937^{* * *}$ & -0.101 \\
\hline 7 & 100-GW & & & & & & & 0.256 & 0.459 & 0.477 & 0.468 & -0.358 & -0.234 & -0.190 \\
\hline 8 & FLA & & & & & & & & 0.197 & 0.599 & $0.785 *$ & 0.361 & $0.845^{*}$ & -0.436 \\
\hline 9 & FLAr & & & & & & & & & -0.139 & 0.686 & $-0.802^{*}$ & 0.017 & $-0.739 *$ \\
\hline 10 & 2nd LA & & & & & & & & & & 0.186 & 0.287 & 0.472 & 0.127 \\
\hline 11 & 2nd LAr & & & & & & & & & & & -0.153 & 0.488 & $-0.775^{*}$ \\
\hline 12 & HI & & & & & & & & & & & & 0.424 & 0.464 \\
\hline 13 & PPCG & & & & & & & & & & & & & -0.343 \\
\hline 14 & LAR & & & & & & & & & & & & & \\
\hline 15 & LWR & & & & & & & & & & & & & \\
\hline 16 & SLW & & & & & & & & & & & & & \\
\hline 17 & SLA & & & & & & & & & & & & & \\
\hline 18 & LAI & & & & & & & & & & & & & \\
\hline 19 & LAD & & & & & & & & & & & & & \\
\hline 20 & RLGR & & & & & & & & & & & & & \\
\hline 21 & RGR & & & & & & & & & & & & & \\
\hline 22 & NAR & & & & & & & & & & & & & \\
\hline 23 & CHL & & & & & & & & & & & & & \\
\hline 24 & $\mathbf{N}_{2}$ & & & & & & & & & & & & & \\
\hline 25 & CAR & & & & & & & & & & & & & \\
\hline 26 & GYP & & & & & & & & & & & & & \\
\hline
\end{tabular}


Table.2 Path Co-efficient analysis of direct (diagonal) and indirect effects of various traits on grain yield per plant

\begin{tabular}{|c|c|c|c|c|c|c|c|c|c|c|c|c|}
\hline Sl.No. & Characters & FD & PH & PL & PN & FGN & F\% & 100-GW & FLA & FLAr & 2nd LAr & HI \\
\hline 1 & FD & -0.795 & 0.534 & 0.062 & -0.123 & -0.177 & -1.487 & 0.726 & 0.830 & -0.733 & 0.434 & 0.017 \\
\hline 2 & PH & -0.126 & 3.355 & 0.038 & -0.431 & -0.109 & 0.563 & -0.558 & 0.514 & -1.524 & 1.147 & 0.040 \\
\hline 3 & PL & 0.359 & -0.923 & -0.138 & 0.233 & 0.227 & -0.179 & -0.063 & -0.741 & 0.519 & -0.624 & -0.016 \\
\hline 4 & PN & 0.165 & -2.426 & -0.054 & 0.595 & 0.316 & 0.654 & 0.870 & -2.430 & 1.344 & -1.557 & -0.019 \\
\hline 5 & FGN & -0.243 & 0.631 & 0.054 & -0.324 & -0.582 & -1.580 & 0.192 & 2.756 & 0.018 & 1.057 & -0.039 \\
\hline 6 & F\% & -0.456 & -0.728 & -0.009 & -0.150 & -0.354 & -2.595 & 0.411 & 2.426 & 0.038 & 0.537 & -0.030 \\
\hline 7 & 100-GW & 0.401 & 1.299 & -0.006 & -0.360 & 0.077 & 0.739 & -1.441 & 0.843 & -0.833 & 0.741 & 0.025 \\
\hline 8 & FLA & -0.200 & 0.523 & 0.031 & -0.439 & -0.487 & -1.912 & -0.369 & 3.292 & -0.357 & 1.244 & -0.026 \\
\hline 9 & FLAr & -0.321 & 2.819 & 0.039 & -0.441 & 0.006 & 0.054 & -0.661 & 0.649 & -1.814 & 1.087 & 0.057 \\
\hline 10 & 2nd LA & 0.139 & -1.443 & -0.018 & -0.154 & -0.099 & -1.220 & -0.687 & 1.972 & 0.252 & 0.295 & -0.020 \\
\hline 11 & 2nd LAr & -0.218 & 2.429 & 0.054 & -0.585 & -0.388 & -0.880 & -0.674 & 2.584 & -1.245 & 1.584 & 0.011 \\
\hline 12 & HI & 0.196 & -1.882 & -0.032 & 0.158 & -0.320 & -1.105 & 0.516 & 1.188 & 1.455 & -0.242 & -0.071 \\
\hline 13 & PPCG & -0.484 & -0.453 & 0.035 & -0.239 & -0.453 & -2.431 & 0.337 & 2.782 & -0.031 & 0.773 & -0.030 \\
\hline 14 & LAR & 0.458 & -2.312 & -0.113 & 0.460 & 0.265 & 0.262 & 0.274 & -1.435 & 1.341 & -1.228 & -0.033 \\
\hline 15 & LWR & -0.116 & 0.564 & 0.023 & -0.421 & -0.090 & -0.973 & -0.993 & 2.110 & -0.960 & 0.960 & 0.020 \\
\hline 16 & SLW & -0.443 & 2.376 & 0.098 & -0.498 & -0.202 & -0.317 & -0.497 & 1.501 & -1.535 & 1.278 & 0.041 \\
\hline 17 & SLA & 0.415 & -2.040 & -0.102 & 0.508 & 0.245 & 0.519 & 0.534 & -1.840 & 1.392 & -1.302 & -0.033 \\
\hline 18 & LAI & 0.085 & -0.362 & -0.081 & 0.241 & 0.525 & 0.727 & -0.160 & -2.094 & -0.316 & -0.798 & 0.039 \\
\hline 19 & LAD & -0.028 & 0.396 & -0.037 & -0.295 & 0.084 & -0.874 & -0.957 & 1.346 & -0.925 & 0.611 & 0.025 \\
\hline 20 & NAR & -0.583 & -0.674 & 0.073 & -0.055 & -0.412 & -1.793 & 0.902 & 1.649 & 0.281 & 0.339 & -0.029 \\
\hline 21 & CHL & -0.315 & -1.986 & 0.046 & 0.254 & -0.244 & -1.043 & 1.158 & 0.326 & 1.147 & -0.494 & -0.043 \\
\hline 22 & $\mathbf{N}_{2}$ & 0.118 & -1.815 & -0.050 & 0.564 & 0.229 & 0.662 & 1.046 & -2.387 & 1.266 & -1.400 & -0.022 \\
\hline 23 & CAR & -0.330 & 2.413 & 0.113 & -0.378 & -0.317 & 0.189 & -0.046 & 1.021 & -0.976 & 1.082 & 0.019 \\
\hline
\end{tabular}

\begin{tabular}{|c|c|c|c|c|c|c|c|c|c|c|c|c|c|}
\hline Sl.No. & Characters & PPCG & LAR & LWR & SLW & SLA & LAI & LAD & NAR & CHL & $\mathbf{N}_{2}$ & CAR & $\begin{array}{c}\text { Correlation } \\
\text { with yield }\end{array}$ \\
\hline 1 & FD & 0.163 & 0.336 & 0.197 & -0.366 & 1.007 & -0.088 & 0.035 & 0.329 & 0.678 & -0.507 & -0.968 & 0.088 \\
\hline 2 & PH & -0.036 & 0.402 & 0.227 & -0.477 & 1.173 & -0.089 & 0.117 & -0.090 & -1.014 & -1.852 & -1.677 & -0.444 \\
\hline 3 & PL & -0.069 & -0.479 & -0.223 & 0.479 & -1.426 & 0.486 & 0.269 & -0.237 & -0.574 & 1.239 & 1.911 & 0.041 \\
\hline 4 & PN & -0.108 & -0.451 & -0.953 & 0.563 & -1.646 & 0.334 & -0.492 & -0.042 & 0.729 & 3.245 & 1.481 & 0.098 \\
\hline 5 & FGN & 0.209 & 0.265 & 0.208 & -0.234 & 0.814 & -0.746 & -0.144 & 0.318 & 0.717 & -1.349 & -1.272 & 0.742 \\
\hline 6 & F\% & 0.252 & 0.059 & 0.506 & -0.082 & 0.386 & -0.231 & 0.335 & 0.310 & 0.688 & -0.873 & 0.170 & 0.649 \\
\hline 7 & 100-GW & -0.063 & 0.111 & 0.929 & -0.232 & 0.716 & 0.092 & 0.661 & -0.281 & -1.377 & -2.485 & -0.075 & -0.477 \\
\hline 8 & FLA & 0.227 & 0.254 & 0.864 & -0.307 & 1.079 & -0.526 & 0.407 & 0.225 & 0.170 & -2.482 & -0.723 & 0.538 \\
\hline 9 & FLAr & 0.005 & 0.431 & 0.713 & -0.570 & 1.480 & 0.144 & 0.507 & -0.069 & -1.082 & -2.389 & -1.255 & -0.625 \\
\hline 10 & 2nd LA & 0.127 & -0.074 & 1.026 & 0.002 & 0.274 & -0.067 & 0.682 & 0.023 & -0.067 & -1.694 & 0.922 & 0.255 \\
\hline 11 & 2nd LAr & 0.131 & 0.452 & 0.817 & -0.543 & 1.586 & -0.417 & 0.384 & 0.096 & -0.534 & -3.026 & -1.593 & 0.042 \\
\hline 12 & HI & 0.114 & -0.271 & -0.390 & 0.387 & -0.909 & -0.456 & -0.352 & 0.182 & 1.044 & 1.075 & 0.628 & 0.937 \\
\hline 13 & PPCG & 0.269 & 0.200 & 0.579 & -0.217 & 0.813 & -0.451 & 0.224 & 0.371 & 0.856 & -1.349 & -0.462 & 0.679 \\
\hline 14 & LAR & -0.092 & -0.583 & -0.570 & 0.655 & -1.849 & 0.368 & -0.098 & -0.173 & 0.116 & 2.365 & 2.116 & 0.204 \\
\hline 15 & LWR & 0.115 & 0.247 & 1.349 & -0.396 & 1.285 & 0.001 & 0.880 & -0.004 & -0.676 & -3.033 & -0.147 & -0.190 \\
\hline 16 & SLW & 0.087 & 0.567 & 0.793 & -0.673 & 1.901 & -0.236 & 0.318 & 0.115 & -0.413 & -2.735 & -1.862 & -0.326 \\
\hline 17 & SLA & -0.113 & -0.559 & -0.898 & 0.663 & -1.930 & 0.306 & -0.354 & -0.145 & 0.289 & 2.896 & 1.787 & 0.226 \\
\hline 18 & LAI & -0.147 & -0.260 & 0.001 & 0.193 & -0.714 & 0.827 & 0.396 & -0.286 & -0.863 & 0.931 & 1.453 & -0.670 \\
\hline 19 & LAD & 0.060 & 0.057 & 1.192 & -0.216 & 0.687 & 0.329 & 0.995 & -0.129 & -1.026 & -2.328 & 0.649 & -0.323 \\
\hline 20 & NAR & 0.223 & 0.226 & -0.011 & -0.172 & 0.625 & -0.527 & -0.286 & 0.448 & 1.421 & -0.079 & -0.896 & 0.675 \\
\hline 21 & CHL & 0.134 & -0.040 & -0.533 & 0.162 & -0.326 & -0.417 & -0.596 & 0.372 & 1.712 & 1.571 & -0.119 & 0.724 \\
\hline 22 & $\mathbf{N}_{2}$ & -0.106 & -0.403 & -1.195 & 0.538 & -1.633 & 0.225 & -0.677 & -0.010 & 0.786 & 3.423 & 1.062 & 0.179 \\
\hline 23 & CAR & 0.053 & 0.529 & 0.085 & -0.537 & 1.478 & -0.515 & -0.277 & 0.172 & 0.087 & -1.558 & -2.333 & -0.059 \\
\hline
\end{tabular}

FD: Days to Flowering, PH: Plant Height, PL: Panicle Length, PN: Panicle Number, FGN: Fertile Grain Number, F\%: Fertility Percentage, 100GW: 100 Grain Weight, FLA: Flag Leaf Angle, FLAr: Flag Leaf Area, 2ndLA: Second Leaf Angle, 2ndLAr: Second Leaf Area, HI: Harvest Index, PPCG: Post-heading photosynthate contribution to grain, LAR: Leaf Area Ratio, LWR: Leaf Weight Ratio, SLW: Specific leaf weight, SLA: Specific Leaf Area, LAI: Leaf Area Index, LAD: Leaf Area Duration, RLGR: Relative Leaf Growth Rate, RGR: Relative Growth Rate, NAR: Net Assimilation Rate, CHL: Chlorophyll Content, $\mathrm{N}_{2}$ : Nitrogen content, CAR: Carbohydrate Content, GYP: Grain yield per Plant 
The other traits like, harvest index, panicle length, number of fertile grains per panicle, leaf area ratio, specific leaf weight, days to $50 \%$ flowering, 100- grain weight, flag leaf area, specific leaf area, carbohydrate content and fertility percentage exhibited negative direct effect on grain yield.

Out of the different characters under study the traits like fertility percentage, carbohydrate content, specific leaf area, number of fertile grains per panicle, flag leaf area, harvest index, 100-grain weight, days to $50 \%$ flowering, leaf area ratio, post flowering photosynthetic contribution to grain yield, specific leaf weight, net assimilation rate, panicle length and $2^{\text {nd }}$ leaf angle exerted higher indirect effect on yield via other traits.

Thus the foregoing observations on direct and indirect effects, the traits like days to $50 \%$ flowering, plant height, panicle number, number of fertile grains per panicle, fertility percentage, 100-grain weight, flag leaf angle, flag leaf area, $2^{\text {nd }}$ leaf area, harvest index, post flowering photosynthetic contribution to grain yield, leaf area ratio, leaf weight ratio, specific leaf area, leaf area index, leaf area duration, net assimilation rate, chlorophyll content, nitrogen content and carbohydrate content are considered as important selection criteria for the realization of high and stable yields.

The correlation coefficient between the characters like panicle number, 100-grain weight, flag leaf angle, flag leaf area, $2^{\text {nd }}$ leaf area, $2^{\text {nd }}$ leaf angle, post flowering photosynthetic contribution to grain yield, net assimilation rate, specific leaf weight, chlorophyll content, nitrogen content and carbohydrate content more or less similar direct effect indicating that correlation explains the true relationship and therefore direct selection through these traits would be effective.

\section{References}

Dhurai S .2014. Correlation and path coefficient analysis for yield and quality traits under organic fertilizer management in rice (Oryza sativa L.), Electronic Journal of Plant Breeding, 5(3): 581-587.

FuJin-Dong, Yong-Feng Yan, Byun-Woo Lee 2009, Physiological Characteristics of a Functional Stay-Green Rice“'SNU-SG1” during Grain-Filling Period. J. Crop Sci. Biotech. 2009 (March) 12 (1): 47 -52.

Gour. L, Koutu. GK, Singh SK, Patel DD, Shrivastava A and Singh Y, 2017. Genetic variability, correlation and path analyses for selection in elite breeding materials of rice (Oryza sativa L.) genotypes in Madhya Pradesh, The Pharma Innovation Journal, 6(11): 693696

Jennings PR, Berio LE, Torres E, Corredor E (2012) A breeding strategy to increase rice yield potential. FLAR, Colombia

Joshi AK, Kumari M, Singh VP, Reddy CM, Kumar S, Rane J, Chand R (2007) Stay green trait: variation, inheritance and its association with spot blotch resistance in spring wheat (Triticum aestivum L.). Euphytica (2007) 153: 59-71.

Kar R.K (2015). Genetic basis of yield variations in lowland rice with tolerance to submergence and stagnant flooding. (Unpublished, M.Sc. (Ag.) Thesis). Orissa University of Agriculture and Technology, Bhubaneswar, Odisha.

Lu, Q.T.; Lu C.M.; Zhang, J.H.; Kuang, T.Y. Photosynthesis and chlorophyll $a$ fluorescence during flag leaf senescence of field-grown wheat plants. Journal of Plant Physiology, v.159, p.1173-1178, 2002.

Lingaraja.L, Mohammad Shafiqurrahaman, Sriharsha V. P. and Suresh B. G. 2015. Estimation of genetic variability, direct and indirect effects of yield contributing 
traits on grain yield in aerobic rice (Oryza sativa L.) germplasm, The Ecoscan, $\quad 9(1 \& 2): \quad 357-361$, (Supplement on Rice).

Luo P, Ren Z, Wu X, Zhang H, Zhang H, Feng J. 2006. Structural and biochemical mechanism responsible for the stay-green phenotype in common wheat. Chinese Sci. Bull. 51: 2595-
2603.

Naseer Sarfraz, Kashif Muhammad, Ahmad Hafiz Muhammad, Iqbal Muhammad Sarfaraz and Ali Qurban, 2015. Estimation of genetic association among yield contributing traits in aromatic and non-aromatic rice (Oryza sativa L) cultivars, Life Science Journal 2015;12(4s): 68-73.

\section{How to cite this article:}

Suman Kumari Nayak, R.K. Panda and Das, S.R. 2020. Correlation and Path Analysis of Physiological and Genetic Traits in Stay Green Rice (Oryza sativa L.) Genotypes. Int.J.Curr.Microbiol.App.Sci. 9(07): 2023-2029. doi: https://doi.org/10.20546/ijcmas.2020.907.233 